\title{
Fragmentation Characteristic of Glutathione Conjugates Activated by High-Energy Collisions
}

\author{
Constance M. Murphy and Catherine Fenselau \\ Department of Chemistry and Biochemistry, University of Maryland Baltimore County, \\ Baltimore, Maryland, USA
}

\section{Peter L. Gutierrez}

The University of Maryland Cancer Center, University of Maryland at Baltimore, Baltimore, Maryland, USA

\begin{abstract}
Product ion spectra of fifteen monoglutathione and diglutathione conjugates have been measured using activation by $6000-\mathrm{eV}$ collisions with helium in the third field-free region of a four-sector tandem mass spectrometer of EBEB configuration. Fragmentation patterns in the cation spectra have been analyzed for decompositions of the glutathione moiety that would permit recognition of an unknown as a glutathione conjugate. Five spectra from an earlier study of high-energy collisional activation on a BEEB four-sector instrument have also been included in this analysis. A suite of appropriate ions was found to occur consistently, including ions of $\mathrm{m} / \mathrm{z} 307$ comprising the glutathione tripeptide and the complementary ion $[\mathrm{MH}-307]^{+}$or the ion radical $[\mathrm{MH}-306]^{+*}$. (J Am Soc Mass Spectrom 1992, 3, 815-822)
\end{abstract}

$\mathrm{T}$ The chemical reactions of glutathione, including those enzymatically catalyzed, are important components of the defense system of every cell against xenobiotic insult. Conjugation with glutathione can also inactivate therapeutic agents, and is involved in regulation of endogenous substrates.

Mass spectral analysis of this important and widely encountered family of metabolites was not satisfactorily achieved before the advent of desorption techniques. Some successful applications have been reported using field desorption (FD); however, derivatization appears to provide more reliable FD results [1]. Subsequently fast atom bombardment (FAB) [2], plasma desorption [3], and electron ionization following laser ablation [4] have been successfully used in this and other laboratories to analyze intact glutathione conjugates. In cation spectra, abundant protonated and/or natriated molecular ions are usually observed, along with class-characteristic ions of $\mathrm{m} / \mathrm{z}$ 306,307 , or 308 , corresponding to glutathione, molecular weight $307 \mathrm{Da}$.

Tandem mass spectrometry has also been evaluated for the dual purposes of working with incompletely purified metabolites and of inducing more extensive fragmentation by collisional activation. This work has

Address reprint requests to Catherine Fenselau, Department of Chemistry and Biochemistry, Uruversity of Maryland Baltimore County, 5401 Wilkens Avenue, Baltimore, MD 21228 mainly been carried out with FAB, although FD was used in one early report [5]. Spectra from more than a dozen glutathione conjugates activated by low-energy collisions have been published [6-11] and another dozen have been analyzed by high-energy collisional activation [12-19]. Derivatized glutathione adducts have also been characterized by collisionally induced dissociation (CID) [11, 20-24].

Fragmentation is indeed found to be enhanced by collisional activation, and, in general, fragmentation patterns produced by high- and low-energy collisions have more in common than not. However, the recognition of class-characteristic ions in either low- or highenergy CID experiments has been uncertain. This has led laboratories using quadrupole-based tandem instruments to evaluate detection of constant neutral losses as an alternative strategy for recognizing glutathione conjugates. Straub [6] proposed that this class could be recognized by the neutral loss of $275 \mathrm{Da}$ under collisional activation at $29 \mathrm{eV}$. Heeremans et al. [7] reported the loss of $146 \mathrm{Da}$ using $45-\mathrm{eV}$ collisions. Haroldson et al. [8], analyzing low-energy collisions of seven different conjugates, observed both of these losses, as well as loss of $129 \mathrm{Da}$. None of these losses was observed consistently in all seven spectra, although $[\mathrm{M}+\mathrm{H}-232]^{+}$ions were detected in the product ion scans of all seven samples, formed by multistep processes. This latter group has favored the strategy of constant neutral loss scanning for $129 \mathrm{Da}$ [10]. It should be noted that these four dissociations, 
like most induced by low-energy collisions, involve formation of even-electron or closed-shell products.

High-energy collisions have also been used to activate glutathione conjugate ions. Lay et al. [13] reported the elimination of neutral fragments of 75 and $129 \mathrm{Da}$ from three protonated conjugates activated by collisions with helium at $8000 \mathrm{eV}$ in a three-sector instrument. A series of cation spectra have been reported, measured on the BEEB four-sector instrument at the National Institute of Environmental Health Sciences [14-16], which record losses of neutral fragments of masses 75 and 129, and also formation of ions of mass 306 or 307. Deterding and co-workers [16] have analyzed both cation and anion spectra of glutathione conjugates measured on that instrument. Even electron ions of $m / z 306$ or the ion radical, $m / z$ 307, were observed in fragmentation of all five of the protonated conjugates studied. $[\mathrm{MH}-129]^{+}$ions were present in four spectra. Formation of $[\mathrm{MH}-75]^{+}$ions, of [MH -232$]^{+}$ions, and of ion radicals of $\mathrm{m} / \mathrm{z} 130$ were each detected in three cases. Ion radicals are commonly generated in high-energy collisional activation from protonated (even-electron) molecules.

The variations observed in the fragmentation patterns in this combined body of literature are assumed to reflect the chemical natures of the conjugation partners of glutathione, and also the differences in fragmentation processes induced by high-and low-energy collisions. In the present study we summarize highenergy CID studies on 15 additional protonated glutathione conjugates and integrate our results with the Deterding study [16] to assess general patterns of fragmentation in high-energy CID.

\section{Experimental}

Mass Spectrometry. Measurements were obtained on a JEOL (Tokyo, Japan) HX110/HX110 four-sector mass spectrometer (EBEB, where E represents an electric sector and B a magnetic sector). Conventional magnetic scans were obtained with the first two sectors (EB), using a JEOL FAB gun and a JEOL DA5000 data system. The FAB gun was operated at $6 \mathrm{kV}$ with xenon. The resolution was 3000 .

Tandem measurements were obtained by utilizing all four sectors (EBEB) on the JEOL HX110/HX110 instrument. Product ions were analyzed with MS-II, following mass selection of the precursor ions by MS-I and collisional activation. The resolution in both MS-I and MS-II was 1000 . Helium was used for CID in the third field-free region, at pressures sufficient to attenuate the precursor ion by $80 \%$. The accelerating voltage in MS-I was $10 \mathrm{kV}$, and the collision cell was floated to $4 \mathrm{kV}$. Spectra were plotted with the most abundant fragment ion at or near $100 \%$ relative abundance.

Samples (1-10 ng) were dissolved in methanol (Fisher Scientific Co., Fair Lawn, NJ) or a suitable methanol/water mixture and added to a matrix on the probe tip. The matrix was either monothioglycerol (Sigma Chemical Co., St. Louis, MO) or 3-nitrobenzyl alcohol (Aldrich Chemical Co., Milwaukee, WI).

Glutathione Conjugates. The structures of the ten monoconjugates and five diconjugates studied are shown in Figure 1. Conjugates of phenylglyoxal dithiohemiacetal (II and XIV), dithiomandelic acid (III) and lactoic acid (VII) were synthesized in the laboratory of Dr. Donald Creighton [24, 25]. The glutathione conjugate of epoxy-dimethylbilirubin (VIII) was isolated in the laboratory of Dr. G. Odell, University of Wisconsin at Madison [18, 19]. The monoconjugate of cyclophosphamide (I) and the diconjugate of phosphoramide mustard (XI) were synthesized by $Z$. Yuan [4, 17]. Conjugates of 2,4-nitrobenzene (VI) and styrene oxide (V) were synthesized chemically [2] by $X$. Yu in this laboratory. Monogiutathione and diglutathione conjugates IX, XII, and XIII were formed by chemical reaction of glutathione with diaziquone (2,5-diaziridinyl3,6-bis(carboethoxyamino)-1,4-benzoquinone) (AZQ) [26]. Mixed disulfide conjugates of $\left[\mathrm{Arg}^{8}\right]$-vasopressin (X, XV) were synthesized chemically in this laboratory [27]. Hexyl glutathione (IV) was purchased from Sigma Chemical Co.

\section{Results}

Initially, FAB spectra were obtained for the 15 glutathione conjugates. Protonated molecular ions were observed for all the compounds. These $[\mathrm{M}+\mathrm{H}]^{+}$ions were subsequently fragmented via high-energy collisions with helium. The results of the CID experiments are summarized in Table 1. To facilitate the discussion of glutathione fragmentation, the convention proposed by Gaskell and co-workers [8] has been adapted as summarized in Scheme I. Neither the ionizing proton nor proton transfers are shown.

The ten monoglutathione conjugates studied encompass a variety of chemical structures (Figure 1). Figure 2 presents the CID spectrum of the protonated monoglutathione conjugate of cyclophosphamide (I) as a representative spectrum. Only ions containing ${ }^{35} \mathrm{Cl}$ and ${ }^{12} \mathrm{C}$ were selected by MS-I for activation in this case. There are a number of glutathione-related fragments recorded: $a, b, d, e, f, g, i, k$, and $R$, as listed in Table 1. Additionally, several fragment ions are derived from the drug moiety, including the most abundant, $m / z 211$ [17]. It should be noted that ions of $m / z$ 307 (type i) comprise the glutathione moiety and are ion radicals.

The CID spectrum of compound IX, derived from the antitumor drug $A Z Q$, is shown in Figure 3. As usual, ions containing only ${ }^{12} \mathrm{C}$ were selected by MS-I, and thus ${ }^{13} \mathrm{C}$ isotopic peaks are absent, for example, around the peak at $m / z$ 562. All of the glutathione ions shown in Scheme I are formed (Table 1) except the multistep loss of $232 \mathrm{Da}(\mathrm{g})$. Abundant ions of masses 


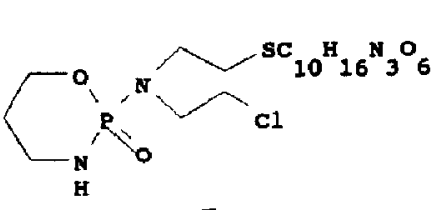

I<smiles>CCCCCC[Se][13CH2][13CH2][13CH3]</smiles>

IV<smiles>[13CH3][13CH2][13CH2]SC(S)C(=O)c1ccccc1</smiles>

I I

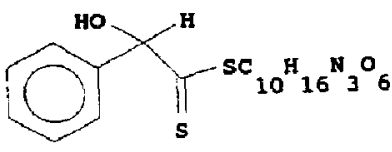

III<smiles>[13CH3]SCC(O)c1ccccc1</smiles>

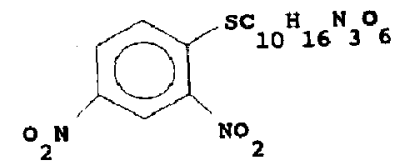

VI

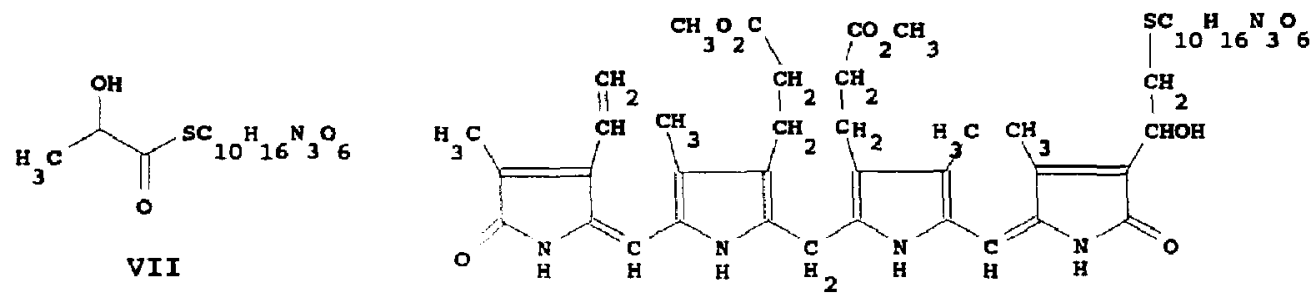

VIII<smiles>CCOC(=O)NC1=C(NCCO)C(=O)C(NCCO)=C(NCCOC(=O)OCC)C1=O</smiles>

Cys-Tyr-Phe-GIn-Asn-Cys-Pro-Arg-Gly

s ${ }^{5 C}{ }_{10}{ }^{H} 16^{N}{ }_{3}{ }_{6}$

$\mathbf{x}$

IX

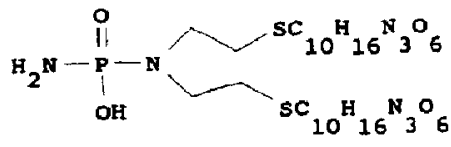

$\mathbf{X I}$

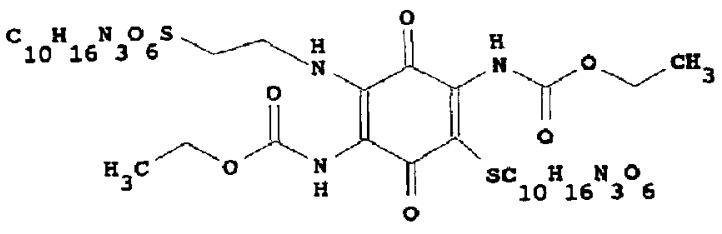

XII

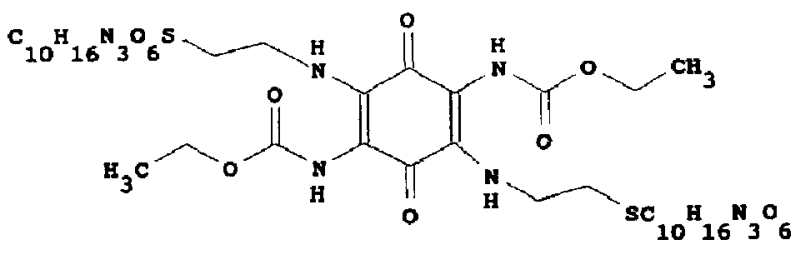

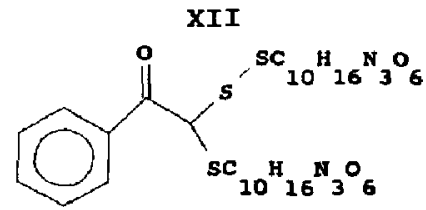

XIV

XIII

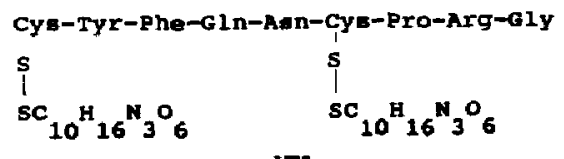

Xv

Figure 1. Structures of glutathione conjugates used in this study. The structure for glutathione, indicated as $\mathrm{SC}_{10} \mathrm{H}_{16} \mathrm{~N}_{3} \mathrm{O}_{6}$ in each conjugate, is shown in Scheme I. 


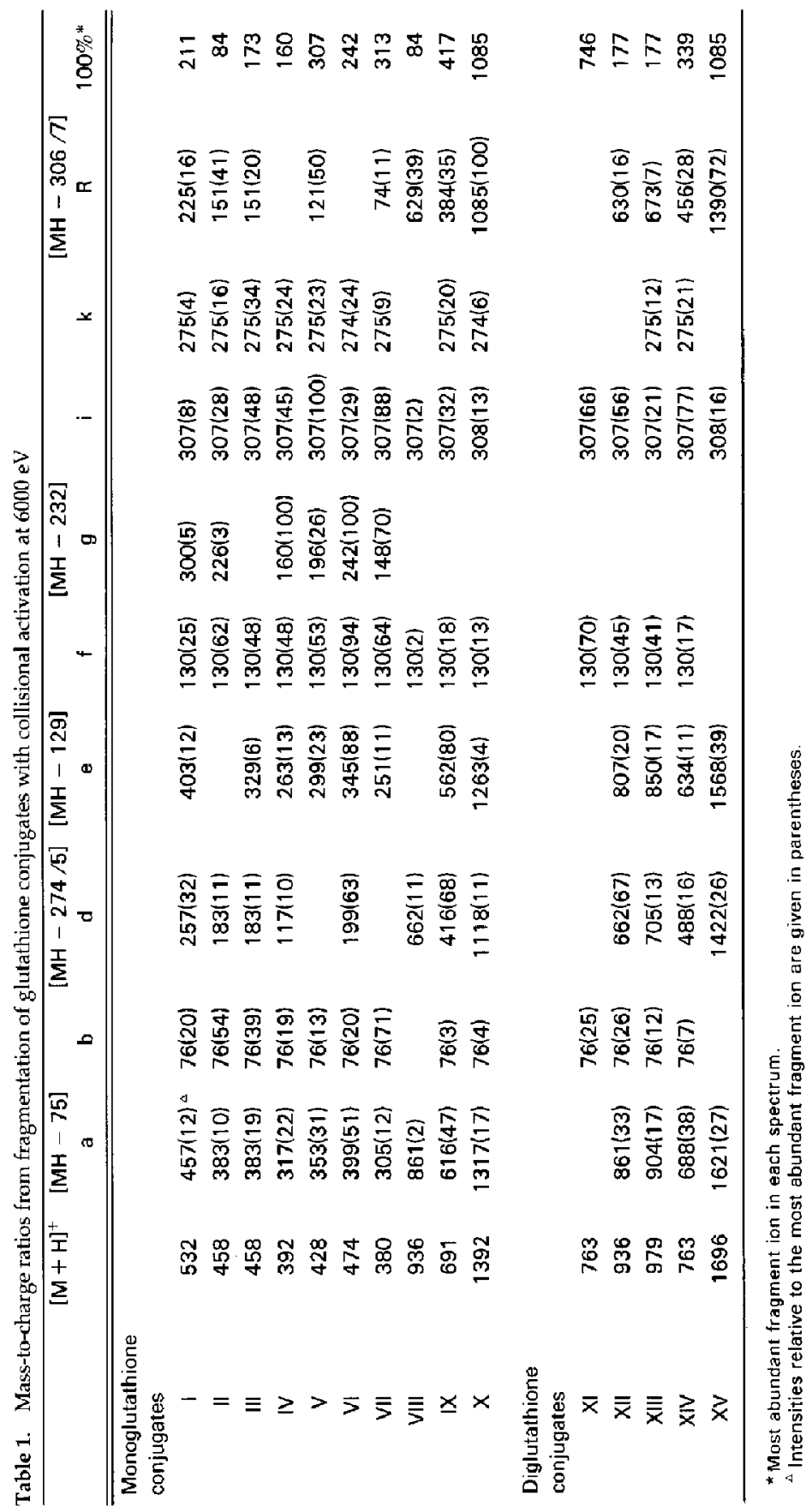




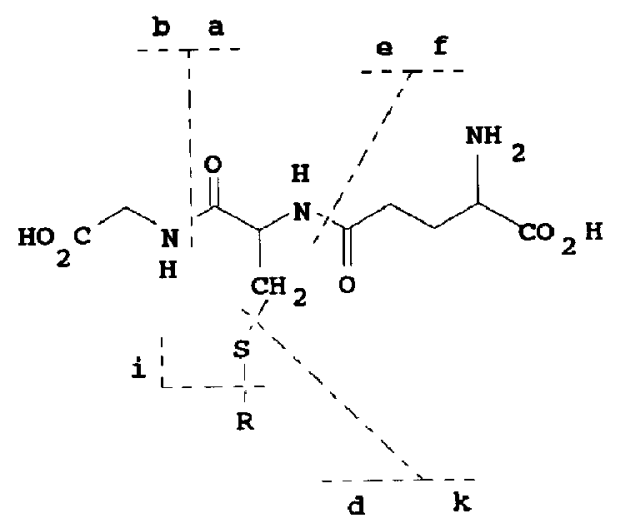

Scheme I

416,417 , and 418 are formed by loss of 275,274 , and 273 Da. These are interpreted to be even-electron ions and ion radicals of the general class $d$, formed by cleavage of a C-S bond, with and without hydrogen transfers. A similar multiplicity of processes is reflected in formation of ions of $m / z 384,385$, and 386 by cleavage of the other C-S bond. Major peaks at $m / z$ 618 and 312 correspond to ions formed by loss of the ethylcarboxyl side chain in the drug moiety.

A spectrum of the mixture of isomeric glutathione conjugates formed from styrene oxide was chosen for presentation in Figure 4, because it has been studied by other laboratories as well [16], and because it is readily available as a standard. All ions summarized in Scheme I and Table 1 are present in medium to high abundance. Notably, the base peak in the spectrum corresponds to the ion radical of glutathione at $m / z$ 307.

CID spectra of five diglutathione conjugates are also summarized in Table 1 . It should be noted that glutathione is conjugated through both thioether and disulfide bonds in this set of samples. In all cases three or more ions from the class-characteristic suite are present, in particular, glutathione ions of $m / z 307$ or 308. In the CID spectrum (shown in Figure 5) of the diconjugate derived from phenylglyoxal dithiohemiacetal (XIV), ions of $m / z 307$, in concert with a suite of other class-characteristic ions (Table 1), reveal the pres-

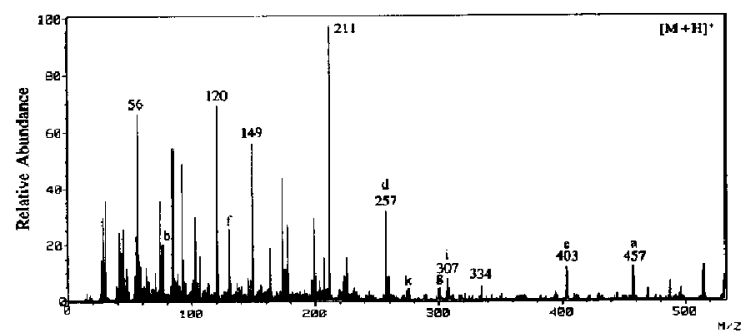

Figure 2. CID spectrum of $I$, the monoglutathione conjugate of cyclophosphamide, $[\mathrm{MH}]^{+}=532 \mathrm{Da}$.

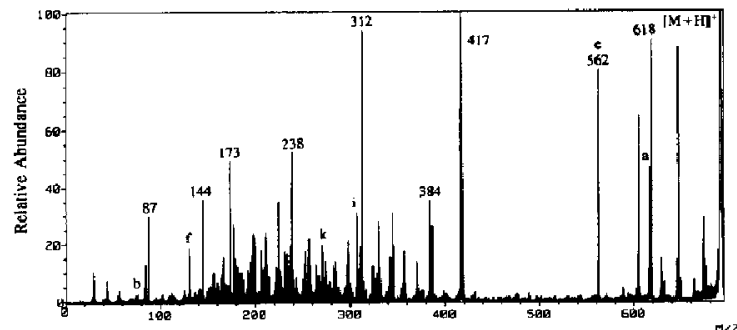

Figure 3. CID spectrum of IX, a monoglutathione conjugate derived from $A Z Q,[M H]^{+}=458 \mathrm{Da}$.

ence of one glutathione moiety. The presence of a second is not obvious from the spectrum, apart from the mass-to-charge ratio of the molecular ion. The most abundant fragment ions have $m / z 339$ and are assigned as $\mathrm{GSSH}^{+}$[27]. The presence of two glutathione units is clearly revealed only in the CID spectrum of diglutathione conjugate $X V$, where sequential losses produce intense peaks at $m / z$ 1390 (MH - 307) and $m / z 1085$ (MH - 612) (Table 1).

Table 1 indicates that, with the exception of conjugate VIII, spectra of the monoglutathione and diglutathione conjugates studied here under high-energy collisional activation exhibit peaks of medium to high intensity at $m / z 307$ or 308 . These peaks correspond to the glutathione moiety and would assist class recognition in an unknown spectrum. In the low-mass range, most of these spectra contain medium intensity peaks corresponding to ions $\mathrm{b}$ of $m / z 76$ comprising glycine from glutathione, and to ions $f$ of $m / z 130$ comprising the glutamyl residue. The complementary processes, loss of 75 (ion a) and loss of 129 (ion e) are frequently. though not always, detected in good abundance (Table 1).

Cleavages of bonds on either side of sulfur were reported more than 25 years ago to be characteristic of thioethers [28]. This bond cleavage was found to be accompanied occasionally by hydrogen transfers; however, the structural requirements for hydrogen transfer have remained elusive. This same situation is observed in the thioether spectra summarized in Table 1 . The $i$ ions formed by scission of a bond to sulfur (Scheme I)

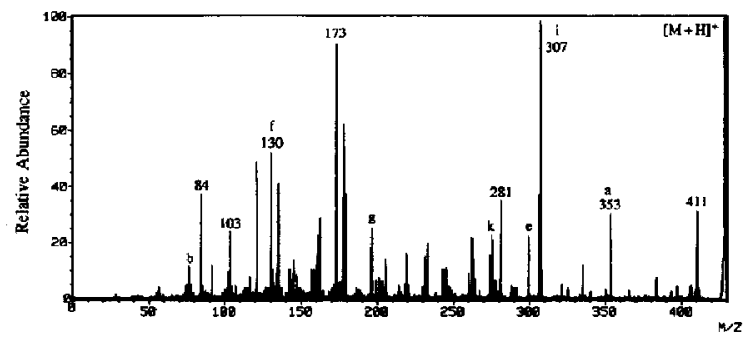

Figure 4. CID spectrum of $\mathrm{V}$, isomeric monoglutathione conjugates of styrene oxide, $[\mathrm{MH}]^{+}=428 \mathrm{Da}$. 


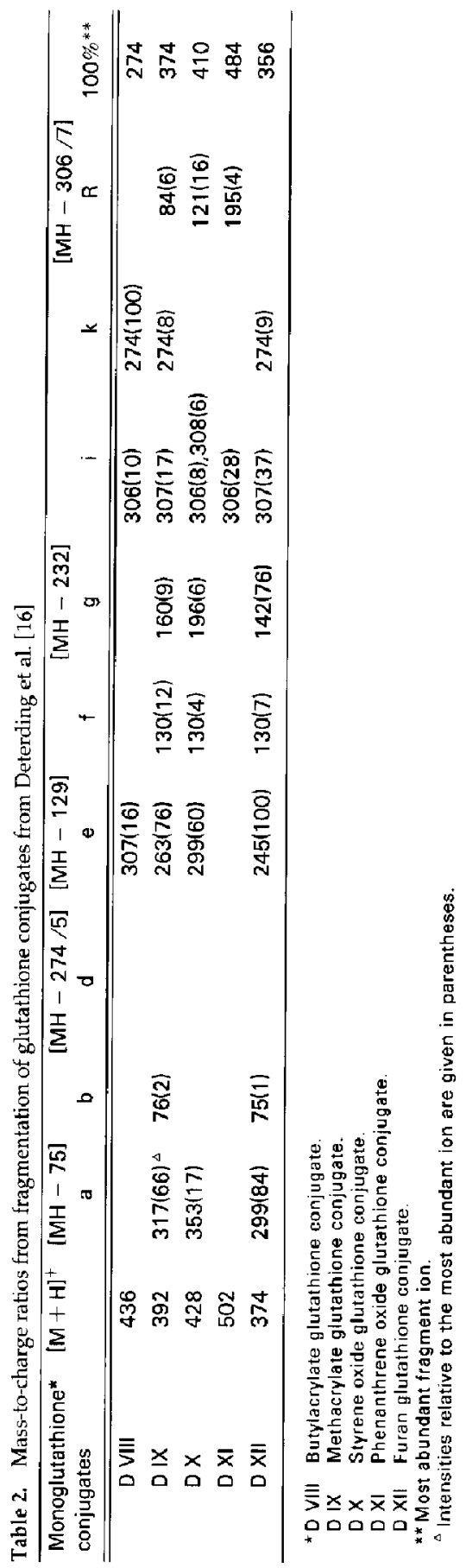




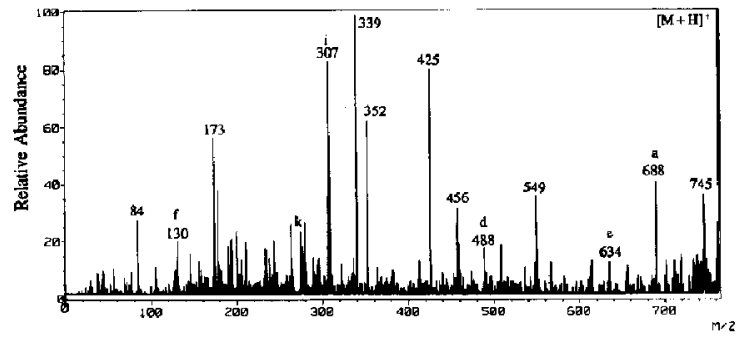

Figure 5. CID spectrum of XIV, the diglutathione conjugate of phenylglyoxal dithiohemiacetal, $[\mathrm{MH}]^{+}=763 \mathrm{Da}$.

appear fairly consistently with $m / z 307$ (hydrogen transfer); however, the complementary $\mathrm{R}$ ions are seen to be formed by loss of either 307 or 306 . Cleavage of the $\mathrm{C}-\mathrm{S}$ bond within the glutathione moiety also produces complementary ions, labeled $\mathrm{d}$ and $\mathrm{k}$ in Scheme I. The $\mathbf{k}$ ions are observed in most cases at $m / z 275$, and their formation is accompanied by hydrogen transfer. However, the complementary d ions listed in Table 1 are formed by either loss of $274 \mathrm{Da}$ (no hydrogen transfer) or $275 \mathrm{Da}$ (hydrogen transfer), varying from one conjugate to another.

The ions formed from compound VIII, the monoglutathione conjugate of epoxy-dimethylbilirubin, include only two of good intensity from the class-characteristic suite (Table 1). Its CID spectrum is dominated by peaks reflecting cleavage at the methylene bridge of bilirubin, namely $m / z 300,313,329,636[18,19]$. Fragmentation characteristic of glutathione occurs on either side of the sulfur atom; however, charge is retained predominately on the bilirubin moiety in fragments $R$ and $d$, reflecting the strong proton affinity of the conjugated tetrapyrrole.

In addition to extensive precedent in the literature, the interpretation of these spectra has been further supported by examination of the collisionally activated decomposition of a number of analogs. These included analogs of VIII in which the epoxide was labeled with ${ }^{18} \mathrm{O}$ [19] or glutamate had been enzymatically removed from glutathione [18], analogs of $I$ and $X I$ in which the mustard moiety carried four ${ }^{2} \mathrm{H}$ labels [17], and analogs of VII in which the glycine carboxy group had been converted to methyl and ethyl esters [24].

\section{Discussion}

The entries in Table 2 marked D VIII through D XII are taken from ref 16, and summarize fragment ions from the high-energy CID spectra of the glutathione conjugates indicated in the footnote. Entries $\mathrm{V}$ in Table 1 and DX in Table 2 are both from glutathione conjugates of styrene oxide with unspecified isomeric composition. There are some general differences between the data in the two tables, notably the presence of more intense $\left[\mathrm{MH}-\mathrm{H}_{2} \mathrm{O}\right]^{+}$peaks in the earlier work and of more abundant low-mass ions in the present work. The latter is likely the result of the use of a floated collision cell, shown to increase transmission of ions with proportionally low kinetic energy [29]. These spectral and instrumental differences would appear to enhance the significance of the common features, most notably the perseverance of glutathionyl ions of $\mathrm{m} / \mathrm{z}$ 306-308.

Examination of the combined measurements from Tables 1 and 2 indicates that the glutathione moiety itself can usually be recognized in product ion scans of cations produced by high-energy collisions. These ions, $m / z 306,307$, or 308, are not usually present in spectra produced by low-energy collisions [6-11]. It will also be productive to look for ions formed by the loss of 306 or $307 \mathrm{Da}$, especially when the conjugate partner is strongly basic. The pair of ions formed by cleavage of the $\mathrm{C}-\mathrm{S}$ bond within glutathione, ions $\mathrm{k}$ and $\mathrm{d}$ in the Gaskell convention [8], can be expected to provide additional indications that glutathione is present. Sulfur bonds in thioether cations are also broken readily in high-energy CID analysis of cysteine, mercapturate, and cysteinylglycine conjugates $[16,18,30]$, and can be expected to characterize other metabolites derived from glutathione conjugates.

\section{Acknowledgments}

This work was supported in part by grants from the National Institutes of Health, GM 21248, and from the National Science Foundation DIR 8914549; the Frank C. Bressler Research Fund; and a Special Research Initiative Support Grant from the University of Maryland Graduate School at Baltimore. It was reported at the 39th Annual Conference of the American Society for Mass Spectrometry, Nashville, TN, May 19-24, 1991.

\section{References}

1. Gaudich, K.; Przybylski, M. Biomed. Mass Spectrom., 1983, 10 , 292-299.

2. Pallante, S. L.; Lisek, C. A.; Dulik, D. M.; Fenselau, C. Drug Metab. Dispos. 1986, 14, 313-318.

3. Fenselau, C.; Wang, R.; Odell, G. B.; Molgilewsky, W. Int. J. Mass Spectrom. Ion Proc. 1989, 92, 289-295.

4. Yuan, Z.; Fenselau, C.; Dulik, D. M.; Martin, W.; Emary, W. B.; Brundrett, R. B.; Colvin, M.; Cotter, R. J. Anal. Chem. $1990,62,868-870$.

5. Nelson, S. D.; Vaishnav, Y.; Kambara, H; Baillie, T. A. Biomed. Mass Spectrom. 1981, 8, 144-151.

6. Straub, K. M., In Mass Spectrometry in Biornedical Research; Gaskell, S. J., Ed.; Wiley: New York, 1986; pp. 115-134.

7. Heeremans, C. E. M.; te Koppele, J. M.; Niessen, W. M. A.; van der Greef, J.; Brussee, I.; La Vos, G. F; ten Noever de Brauw, M. C. Biomed. Environ. Mass Spectrom. 1988, 17, 181-186.

8. Haroldson, P. E.; Reiley, M. H.; Hughes, H.; Gaskell, S. J.; Porter, C. J. Biomed. Environ. Mass Spectrom. 1988, 15, 615-621.

9. Mutlib, A. E.; Talaat, R. E.; Slatter, J. G.; Abbott, F. S. Drug Metab. Dispos. 1990, 18, 1038-1045.

10. Ballard, K. D.; Raftery, M. R.; Jaeschke, H.; Gaskell, S. J. J. Am. Soc. Mass Spectrom. 1991, 2, 55-68.

11. Pearson, P. G.; Howald, W. N.; Nelson, S. D. Anal. Chem. $1990,62,1827-1836$. 
12. Tomer, K. B.; Guenat, C.; Dino, J. J.; Deterding, L. J. Biomed. Environ. Mass Spectrom. 1988, 16, 409-412.

13. Lay, J. O; Potter, D. W.; Hinson, J. A. Biomed. Environ. Mass Spectrom. 1987, 14, 517-521.

14. Stock, B. H.; Bend, J. R.; Eling, T. E. J. Biol. Chem. 1986, 13, 5959-5964.

15. Stock, B. H.; Schreiber, J.; Guenat, C.; Mason, R. P.; Bend J. R.; Eling, T. E. J. Biol. Chem. 1986, 261, 15915-15922.

16. Deterding, L. J.; Srinivas, P.; Mahmood, N. A.; Burka, L. T.; Tomer, K. B. Anal. Biochem. 1989, 183, 94-107.

17. Yuan, Z.; Smith, P. B.; Brundrett, R. B.; Colvin, M.; Fenselau, C. Drug Metab. Dispos. 1991, 19, 625-629.

18. Odell, G. B.; Mogilevsky, W. S.; Smith, P. B. W.; Fenselau, C Molec. Pharm. 1991, 40, 597-605.

19. Shore, L. J.; Mogilevsky, W. S.; Smith, P. B. W.; Fenselau, C.; Odell, G. B. Biochem. Pharmacol. 1991, 42, 1969-1976.

20. Pearson, P. G.; Threadgill, M. D; Howald, W. N.; Baillie, T. A. Biomed. Environ. Mass. Spectrom. 1988, 16, 51-56.

21. Baillie, T. A.; Pearson, P. G.; Rashed, M. S.; Howald, W. N. J. Pharma. Biomed. Anal. 1989, 7, 1351-1360.

22. Pearson, P. G.; Slatter, J. G.; Rashed, M. S.; Han, D.; Grillo,
M. P.; Baillie, T. A. Biochem. Biophys. Res. Commun. 1990, 166 , 245-250.

23. Thomassen, D. T.; Pearson, P. G,; Slattery, I. T.; Nelson, S. D. Drug Metab. Dispos. 1991, 19, 997-1003.

24. Hamilton, D. S.; Creighton, D. J. Biochem. Biophys. Res. Commun. 1992, in press.

25. Li, J.; Guha, M. K.; Creighton, D. J. Biochem. Biophys. Res. Commun. 1991, 181, 657-663.

26. Gutierrez, P. L.; Wilder, P. J.; Biswal, N. Cancer Conmun. 1989, 1, 10-19.

27. Fenselau, C.; Smith, P. B. W. Xenobiotica 1992, in press.

28. Budzikiewicz, H.; Djerassi, C.; Williams, D. H. Mass Spectrometry of Organic Compounds, Holden Day: San Francisco, 1967.

29. Martin, S. A.; Johnson, R. S.; Costello, C. E.; Biemann, K. In The Analysis of Peptides and Proteins by Mass Spectrometry; McNeal, C. J., Ed.; Wiley: New York, 1988, p. 135.

30. Jones, A. D.; Winter, C. K.; Segall, H. J.; Buonarate, M. H. Presented at the 36th Annual Conference on Mass Spectrometry and Allied Topies, San Francisco, CA, June 5-10, 1988. 